\title{
Variation for aggression and response in the competitive interactions of Drosophila melanogaster
}

\author{
Paul Eggleston
}

Department of Genetics, University of Liverpool, P.O. Box 147, Liverpool L69 3BX.

\begin{abstract}
The competitive interactions which take place in genetically heterogeneous cultures of Drosophila melanogaster consist of both intra-genotypic and inter-genotypic effects. If these parameters are quantified separately they can be used to describe the way in which a particular genotype exerts competitive pressure on the one hand and responds to such competitive pressure on the other. The results presented in this paper show that a distinction can be made between the aggression and the response of a series of genotypes derived from the Texas population of Drosophila melanogaster. For most of the mixtures involved in this experiment the deviations from the mean competitive value attributable to aggression $(a)$ and response $(r)$ were quantified and shown to combine additively in their effect on the character $p_{a}$ (the proportion of eggs developing successfully into adult flies, transformed into angles) with no evidence of any interaction. The components $(a)$ and $(r)$ show substantial variation but the lack of any correlation in their distribution suggests that they are separately adjustable by selection. The implications of this result with respect to the action of natural selection within the Texas population is considered.
\end{abstract}

\section{INTRODUCTION}

Recent studies of competition in Drosophila melanogaster have served to emphasise the complexity of the interactions involved. More specifically, the importance of the interaction of closely related genotypes has been demonstrated. These intra-genotypic effects may even dominate those found between unrelated individuals in genetically heterogeneous mixtures (Caligari, 1980). Clearly, any quantitative analysis of these interactions must take both intra and inter-genotypic effects into account in such a way as to allow their comparison and their combination in the prediction of the outcome of a mixture. Such a procedure has been described by Mather and Caligari (1981). Extensions to this analysis have now demonstrated that each individual within a competing group of organisms has a dual role. On the one hand it will be exerting competitive pressure against its contemporaries and on the other it will be responding to the overall competitive pressure it faces in the mixture (Mather and Caligari, 1983). In this way a distinction can be drawn between the aggression and the response of each genotype and these parameters appear to behave as the causal components of competitive interaction. The experiments described in this paper were designed to investigate the variation found for aggression and response within a population of Drosophila melanogaster. The relationship between the components and its implications with respect to genetically segregating populations is considered.

\section{EXPERIMENTAL DETAILS}

The experiment was designed to investigate the competitive interactions which take place between a population of Drosophila melanogaster originating from Texas and a series of inbred lines which were derived from (and are thus representative of) that population. The origin of this material has been described by Linney et al., (1971).

The principle of the approach used has been described by Mather and Caligari (1981). Essentially, two classes of competitive interaction can be recognised. First, the intra-genotypic effect, which is the competition of individuals with like genotype. Secondly, the inter-genotypic effect, which is the competition of individuals with very different genotypes. In genetically heterogeneous mixtures both classes of interaction will be present and the former must be used as a 
yardstick by which the true inter-genotypic effect can be quantified. This form of analysis has now been used for several characters of interest but the character followed in this study was the proportion of eggs successfully developing into adults (character $p$ as described by Caligari, 1980). This character shows, to a reasonable approximation, a linear relationship to culture density, at least over the range of 20 to 180 eggs. Such a relationship facilitates the analysis but is not a pre-requisite. Each value of $p$ was converted into an angle $\left(p_{a}\right)$ using the standard angular transformation (Fisher and Yates, 1963).

Estimation of the intra-genotypic effect was achieved by raising a monoculture density series for each genotype. The slope $\left(b_{m}\right)$ of the regression of $p_{a}$ on culture density is representative of the magnitude of the intra-genotypic competition. Estimation of the inter-genotypic effect was achieved by raising duoculture density series for various genotype mixtures. In this way, the effect of the competition of an associate genotype on an indicator genotype could be measured. Density series were constructed using a substitution design which maintained a reference density of 120 eggs per culture. As eggs of the indicator genotype were removed they were substituted by eggs of the associate genotype and the effect of this substitution on the indicator genotype was noted. The slope $\left(b_{d}\right)$ of the regression of $p_{a}$ on indicator genotype culture density measures two things. First, the effect of the indicator genotype on itself (the intra-genotypic effect) and secondly, the effect of the associate genotype on the indicator genotype (the inter-genotypic effect). From this duoculture slope $\left(b_{d}\right)$ the intra-genotypic effect $\left(b_{m}\right)$ can be subtracted leaving a measure solely of the intergenotypic effect. Thus, as was pointed out by Mather et al., (1982) the magnitude of the intragenotypic effect is measured as the difference between the line of zero relationship and the monoculture slope $\left[\left(b_{0}-b_{m}\right)=-b_{m}\right]$. Similarly, the magnitude of the inter-genotypic effect is measured as the difference between the duoculture and monoculture slopes $\left(b_{d}-b_{m}\right)$. These estimates are referred to as the competitive values.

A pre-requisite for this form of analysis is the need to be able to distinguish the two genotypes involved in each mixture. This was accomplished by the introduction of the $y^{2}$ marker into each of the Texas inbred lines as described by Mather and Caligari (1983). It has now been demonstrated that the introduction of this particular allele has a negligible effect on the competitive interactions although the choice of such markers must be made with care.
The present study consisted of nine series of monocultures, one each for the Texas population and the eight yellow marked inbred lines which were denoted $y^{2} T 1, y^{2} T 5, y^{2} T 15, y^{2} T 17, y^{2} T 19$, $y^{2} T 25, y^{2} T 27$ and $y^{2} T 28$. It should be noted that the Texas population density series was considered as a monoculture despite its implicit genetical heterogeneity. The experiment gave no indication that it could not be treated in this way to obtain an estimate of the inherent competitive characteris. tics of the population. There were eight duoculture series, one for each combination of population with inbred line.

Eggs were collected on blackened starch partitions in $3^{\prime \prime} \times 1^{\prime \prime}(2.5 \times 7.5 \mathrm{~cm})$ vials over a period of two hours at $25 \pm 0.5^{\circ} \mathrm{C}$. This was the minimum time period during which the required quantity of eggs could be collected. Using this technique, heterogeneity of developmental age is kept to a minimum. All cultures were started with a counted number of eggs and conformed to the following density series;

Indicator monocultures $\begin{array}{llll}120 & 90 & 60 & 30\end{array}$

Associate monocultures

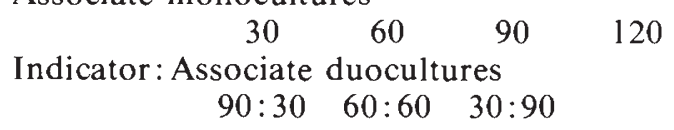

The monocultures with 120 eggs can clearly be considered as the starting point of the duoculture series, that is, a culture where no eggs of the indicator genotype have been substituted. The expression of the character $p_{a}$ at the reference density of 120 eggs is therefore common to both the monoculture and the duoculture slopes.

All cultures were raised in $3^{\prime \prime} \times 1^{\prime \prime}(2.5 \times 7 \cdot 5 \mathrm{~cm})$ vials containing $5 \mathrm{ml}$ of 2 per cent agar onto the surface of which had been dispensed $0.5 \mathrm{ml}$ of live yeast suspension. The original concentration of this suspension was $9 \mathrm{~g}$ dried bakers yeast (Sigma YSC-2) in $100 \mathrm{ml}$ distilled water. Thus each vial had a fixed quantity of $45 \mathrm{mg}$ of live yeast available as food for the newly hathed competing larvae. Eggs were introduced carefully into the surface of this yeast using a sterilised needle.

All cultures were raised at $25 \pm 0.5^{\circ} \mathrm{C}$ in an individually randomised design. The whole experiment was duplicated allowing the difference between duplicate observations to be used as a source of error variation.

\section{AN EXAMPLE}

The analytical procedure can be illustrated by reference to that part of the experiment which 
Table 1 Observed (upper entry) and expected (lower entry) values of $p_{a}$ for each density in the monoculture and duoculture series for the Texas population and $y^{2} T 25$

\begin{tabular}{|c|c|c|c|c|c|c|}
\hline Indicator & Associate & $\begin{array}{l}\text { Density } \\
x\end{array}$ & $\begin{array}{r}30 \\
-90\end{array}$ & $\begin{array}{r}60 \\
-60\end{array}$ & $\begin{array}{r}90 \\
-30\end{array}$ & $\begin{array}{r}120 \\
0\end{array}$ \\
\hline Population & - & & $\begin{array}{l}55 \cdot 5 \\
62 \cdot 4\end{array}$ & $\begin{array}{l}56 \cdot 5 \\
48 \cdot 2\end{array}$ & $\begin{array}{l}39 \cdot 0 \\
34 \cdot 0\end{array}$ & $\begin{array}{l}14 \cdot 0 \\
19 \cdot 8\end{array}$ \\
\hline Population & $y^{2} T 25$ & & $\begin{array}{l}45 \cdot 0 \\
47 \cdot 1\end{array}$ & $\begin{array}{l}42 \cdot 5 \\
38 \cdot 0\end{array}$ & $\begin{array}{l}26 \cdot 5 \\
28 \cdot 9\end{array}$ & - \\
\hline$y^{2} T 25$ & Population & & $\begin{array}{l}5 \cdot 0 \\
5 \cdot 6\end{array}$ & $\begin{array}{l}11 \cdot 0 \\
11 \cdot 7\end{array}$ & $\begin{array}{l}21 \cdot 0 \\
17 \cdot 7\end{array}$ & - \\
\hline$y^{2} T 25$ & - & & $\begin{array}{l}48 \cdot 0 \\
45 \cdot 5\end{array}$ & $\begin{array}{l}36 \cdot 0 \\
38 \cdot 2\end{array}$ & $\begin{array}{l}28 \cdot 0 \\
31 \cdot 0\end{array}$ & $\begin{array}{l}24 \cdot 5 \\
23 \cdot 7\end{array}$ \\
\hline
\end{tabular}

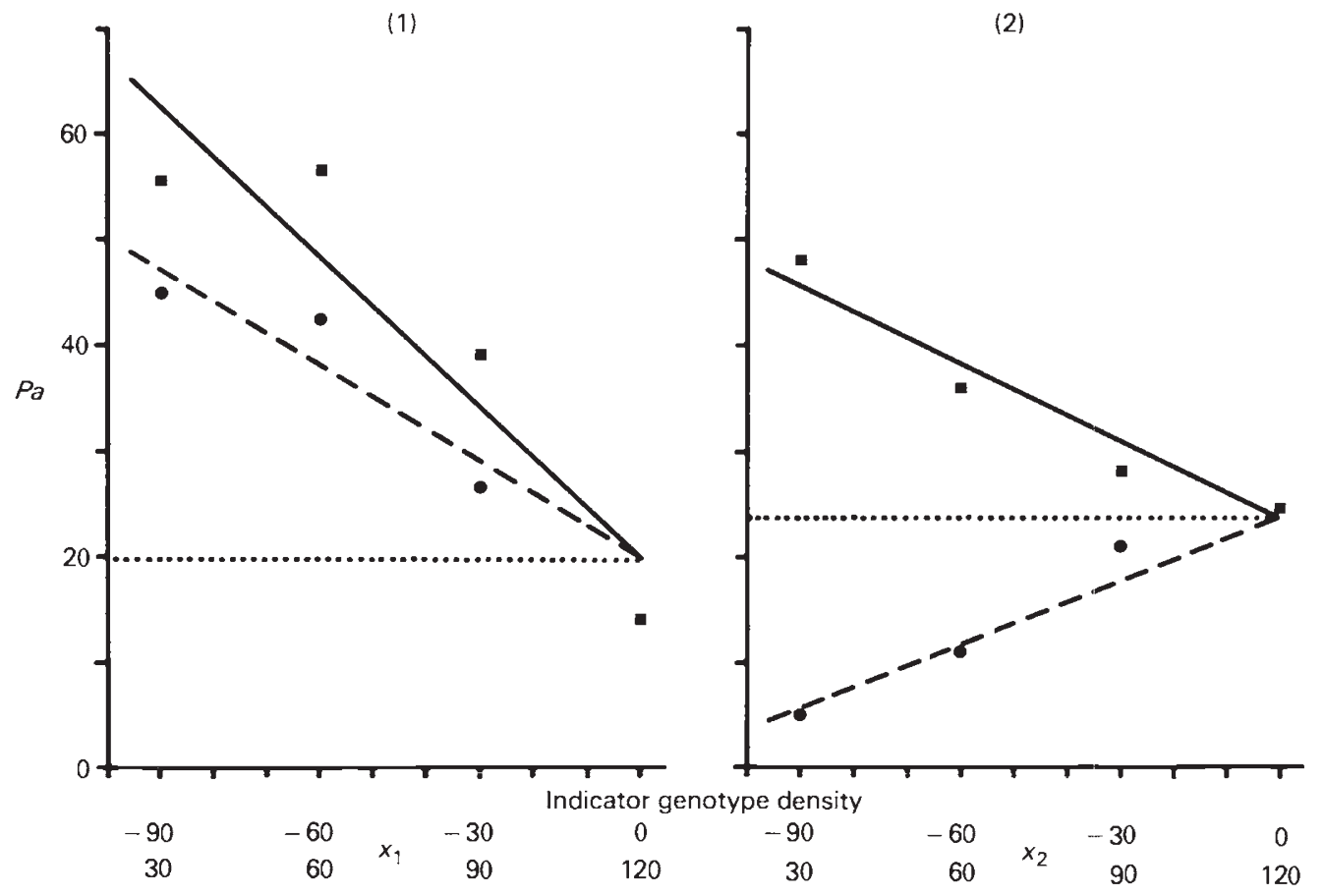

Figure 1 Estimated regression lines relating the character $p_{a}$ to $x$ and therefore to culture density for (1) Texas population as indicator, $y^{2} T 25$ as associate and (2) $y^{2} T 25$ as indicator, Texas population as associate. The monoculture regressions $\left(b_{m}\right)$ are represented by unbroken lines and the duoculture regressions $\left(b_{d}\right)$ by broken lines. The frames of reference are completed by the dotted horizontal lines of zero relationship $\left(b_{0}\right)$. The observed points are shown, monocultures and duocultures being represented by squares and circles respectively.

involved the Texas population and $y^{2} T 25$, the results of which are summarised in table 1 and depicted in fig. 1. The slopes of the regressions of $p_{a}$ on culture density were obtained by least squares estimation as described by Mather and Caligari (1981). These slopes were used to construct lines of best fit to the observed data. The analysis assumes that a linear relationship provides an adequate description of the data and this is tested by calculating the residual variation around the regression lines. If the assumptions of the model are valid, the residual variation should not significantly exceed the error variation to which the original observations were subject. The residual variation around the regression lines for this set of data yields a mean square of 29.753750 for 8 degrees of freedom. The error variation calculated from the difference between duplicate observations amounted to 34.937500 for 12 degrees of freedom. This indicates that a linear relationship provides an adequate description of the data. Estimates for the parameters of interest are given in table 2 . It 
Table 2 Estimates of the regression parameters for the Texas population and $y^{2} T 25$. The expression of the character $p_{a}$ at the reference density of 120 eggs per culture is given as $e$ and the slopes of the monoculture and duoculture lines as $b_{m}$ and $b_{d l}$ respectively

\begin{tabular}{llrrrr}
\hline Indicator & Associate & $e$ & \multicolumn{1}{c}{$b_{d}$} & $\left(-b_{m}\right)$ & $\left(b_{d}-b_{m}\right)$ \\
\hline Population & $y^{2} T 25$ & $19 \cdot 851219$ & -0.302637 & $0 \cdot 473277$ & $0 \cdot 170640$ \\
$y^{2} T 25$ & Population & $23 \cdot 730766$ & $0 \cdot 200917$ & $0 \cdot 241940$ & $0 \cdot 442857$ \\
& & $\pm 4 \cdot 337338$ & \pm 0.081314 & $\pm 0 \cdot 081314$ & \pm 0.074471 \\
\hline
\end{tabular}

can be seen from these results that the magnitude of the intra-genotypic competition between population individuals $\left(-b_{m}\right)$ is 0.473277 .

This, in simple terms, is the amount by which the expression of the character $p_{a}$ increases (beyond its value at the reference density) each time a population egg is removed from the culture and is not substituted. The magnitude of the intergenotypic competition exerted by $y^{2} T 25$ individuals on population individuals $\left(b_{d}-b_{m}\right)$ is $0 \cdot 170640$. This is essentially the change in expression of $p_{a}$ (from its value at the reference density) every time a population egg is removed from the culture and substituted by an egg of the genotype $y^{2} T 25$. Thus if we take as a starting point the value of $p_{a}$ at the reference density (19.851219) removal of a population egg will increase $p_{a}$ by 0.473277 but addition of a $y^{2} T 25$ egg will decrease $p_{a}$ by $\left(b_{d}=0 \cdot 302637\right)$. The difference of $0 \cdot 170640$ is directly attributable to the effect of the associate genotype $y^{2} T 25$. This comparison reflects the fact that the intra-genotypic competition among population individuals is almost three times as strong as the inter-genotypic competition of $y^{2} T 25$ on the population. A similar set of comparisons can be made in respect of the situation where $y^{2} T 25$ is the indicator genotype (table 2). In this case the inter-genotypic competition exerted by the population on $y^{2} T 25\left[\left(b_{d}-b_{m}\right)=0.442857\right]$ is almost twice as strong as the intra-genotypic competition among $y^{2} T 25$ individuals $\left[\left(-b_{m}\right)=0 \cdot 241940\right]$. Clearly, the population individuals play a dominant role in determining the outcome of these mixtures. This is a point to which we shall return.

We can usefully take this analysis a stage further by looking at the way the genotypes exert competitive pressure on the one hand and respond to such pressure on the other. This distinction between pressure (or aggression) and response (or sensitivity) has been inferred previously (Mather et al., 1982; Mather and Caligari, 1983; Breese and Hill, 1973). The estimates of intra-genotypic competition $\left(-b_{m}\right)$, for example, represent the competitive pressure exerted by one genotype upon itself. Similarly, estimates of inter-genotypic competition $\left(b_{d}-b_{m}\right)$ represent the competitive press- ure exerted by the associate on the indicator genotype. In the same way we can consider the response of each indicator genotype to the competitive pressure it faces both from itself in monoculture and from the associate genotype in duoculture. The competitive values derived in this way for the present analysis are given in table 3 .

Table 3 Estimates of the competitive values for the Texas population and $y^{2} T 25$. The lower figure in each of the cells $(\alpha)$ to $(\delta)$ is the expected competitive value assuming that the deviations from the overall mean $(m=0.332178)$ ascribable to aggression $(a)$ and response $(r)$ combine additively in their effect on the character $p_{a}$. The relevant additive model is shown for each cell

\begin{tabular}{|c|c|c|c|c|c|}
\hline \multicolumn{6}{|c|}{ Indicator } \\
\hline & \multicolumn{3}{|c|}{ Population } & $y^{2} T 25$ & \multirow{2}{*}{$\begin{array}{c}\text { Mean } \\
0.458067\end{array}$} \\
\hline Associate & $(\alpha)$ & 0.473277 & $(\beta)$ & 0.442857 & \\
\hline Population & & $\begin{array}{l}0.447847 \\
m+a+r\end{array}$ & & $\begin{array}{l}0 \cdot 468287 \\
m+a-r\end{array}$ & \\
\hline$y^{2} T 25$ & $(\gamma)$ & $0 \cdot 170640$ & $(\delta)$ & $0 \cdot 241940$ & $0 \cdot 206290$ \\
\hline & & $0 \cdot 196070$ & & $0 \cdot 216510$ & \\
\hline & & $m-a+r$ & & $m-a-r$ & \\
\hline Mean & & 0.321958 & & $0 \cdot 342398$ & 0.332178 \\
\hline
\end{tabular}

The total sum of squares of these values can be partitioned into the following three items, each for 1 degree of freedom, using orthogonal functions as described by Mather and Caligari (1983);

$$
\begin{aligned}
R=1 / 4(\alpha-\beta+\gamma-\delta)^{2} & =0.00041779 \\
t_{12} & =0.26, \quad P=0.90-0.70 \\
A=1 / 4(\alpha+\beta-\gamma-\delta)^{2} & =0.06339166 \\
t_{12} & =3.23, \quad P=0.01-0.001 \\
{ }^{\prime} I=1 / 4(\alpha-\beta-\gamma+\delta)^{2} & =0.00258674 \\
t_{12} & =0.65, \quad P=0.70-0.50
\end{aligned}
$$

The error variance of the individual competitive values ( $V c=0.006079$ ) against which $R, A$ and $I$ are tested is derived from the duplicate error variance for 12 degrees of freedom. $R$ tests the significance of the difference in overall response of the two genotypes taken as indicators, $\boldsymbol{A}$ tests 
the significance of the difference in overall aggression of the two genotypes taken as associates and $I$ tests the significance of any interaction between aggression and response. Clearly the genotypes show highly significant differences in the aggression they can exert as associates. There is no indication, however, of any significant difference in response and no evidence of any interaction.

If we assume additivity of aggression and response in their effect on the character $p_{a}$ as in table 3 , we can separate $a$ and $r$ which are the components of the competitive interaction. The appropriate calculations yield;

$$
\begin{array}{rr}
r=1 / 4(\alpha-\beta+\gamma-\delta)= & -0 \cdot 010220 \pm 0 \cdot 038984 \\
a=1 / 4(\alpha+\beta-\gamma-\delta)= & 0 \cdot 125888 \pm 0.038984 \\
i=1 / 4(\alpha-\beta-\gamma+\delta)= & 0 \cdot 025430 \pm 0.038984
\end{array}
$$

where $i$ measures the interaction between $a$ and $r$. If these components are combined additively they yield the expected competitive values in table 3 . The overall fit of the additive model can then be tested as $\chi^{2}{ }_{1}=\Sigma(0-E)^{2} / V c=0.42 ; \quad P=$ $0 \cdot 70-0 \cdot 50$. Thus we have every indication that the components do combine additively. We can therefore conclude that the two genotypes show a difference in response of $2 r=-0 \cdot 020440$ and a difference in aggression of $2 a=0 \cdot 251776$.

\section{RESULTS}

In the first instance the complete analysis as described in the previous section was applied separately to each of the eight combinations of Texas population and inbred line. In each case (with one exception) the relationship between character expression and culture density was adequately described by using linear regressions. The exception was the combination of Texas population and $y^{2} T 5$ where the residual variation after fitting straight lines was significant at the 5 per cent level. This, however, was probably due to the unusually low error variance of 14.068181 rather than to any intrinsic failure of the linear model. Both the residual and the error variances from the eight separate analyses were subjected to Bartletts test of homogeneity. This gave $\chi^{2}{ }_{7}=9 \cdot 36 ; \quad P=$ $0 \cdot 30-0 \cdot 10$ for the error variances and $\chi^{2}{ }_{7}=0 \cdot 795$; $P>0.90$ for the residual variances. In neither case is there any evidence of heterogeneity therefore the data sets were pooled in order to analyse the variation found for aggression and response in the whole experiment.

Using the combined analysis estimates of the regression parameters were obtained as before and used to construct lines of best fit to the data. The overall variation unaccounted for after fitting these straight lines was 29.921000 for 50 degrees of freedom. This does not differ significantly from the error variation of $20 \cdot 847656$ for 64 degrees of freedom to which the original observations were subject. The use which is made of the linear model can therefore be justified. Estimates of the regression parameters are given in tables 4 and 5 .

It is clear from table 4 that the nine genotypes involved show substantial variation in the extent of their intra-genotypic competition. There are two points of particular interest. First, the inbred lines are known to be representative of the range of expression found for many characters within the Texas population and the present results would indicate that this is also true in respect of intragenotypic competition. Secondly, the high level of intra-genotypic competition shown by individuals from the population. We must digress at this stage to consider what is really meant by this statement.

Table 4 Estimates of $e$ (the value of $p_{a}$ at the reference density of 120 eggs per culture) and $\left(-b_{m}\right)$ the magnitude of the intra-genotypic competitive effect for each of the nine genotypes in the experiment. The significance of the deviation of the estimates from zero (tested as normal deviates) is given (NS, $P>0.05 ; *, P=0.05-0.01 ; * *, P=0.01-0.001 ; * * *, P<0.001)$

\begin{tabular}{lccrl}
\hline Genotype & $e$ & $P$ & $\left(-b_{m}\right)$ & $P$ \\
\hline Population & $27 \cdot 470627 \pm 2 \cdot 071721$ & $* * *$ & $0 \cdot 365772 \pm 0.050306$ & $* * *$ \\
$y^{2} T 1$ & $19 \cdot 809409 \pm 3 \cdot 350419$ & $* * *$ & $0 \cdot 102831 \pm 0 \cdot 062812$ & NS \\
$y^{2} T 5$ & $28 \cdot 769228 \pm 3 \cdot 350419$ & $* * *$ & $0 \cdot 168771 \pm 0.062812$ & $* *$ \\
$y^{2} T 15$ & $28 \cdot 192305 \pm 3 \cdot 350419$ & $* * *$ & $0 \cdot 067489 \pm 0.062812$ & NS \\
$y^{2} T 17$ & $29 \cdot 576921 \pm 3 \cdot 350419$ & $* * *$ & $-0 \cdot 016576 \pm 0.062812$ & NS \\
$y^{2} T 19$ & $18 \cdot 384614 \pm 3 \cdot 350419$ & $* * *$ & $-0.036448 \pm 0.062812$ & NS \\
$y^{2} T 25$ & $23 \cdot 730766 \pm 3 \cdot 350419$ & $* * *$ & $0 \cdot 241940 \pm 0.062812$ & $* * *$ \\
$y^{2} T 27$ & $24 \cdot 923074 \pm 3 \cdot 350419$ & $* * *$ & $0 \cdot 170145 \pm 0.062812$ & $* *$ \\
$y^{2} T 28$ & $19 \cdot 115382 \pm 3 \cdot 350419$ & $* * *$ & $0 \cdot 163827 \pm 0.062812$ & $* *$ \\
\hline
\end{tabular}


Clearly, for all of the inbred lines (which have a fixed genotype) the term intra-genotypic competition has a precise meaning, namely the competitive interaction of individuals with the same genotype. With respect to the population, however, this term has been used more loosely to describe its inherent competitive characteristics in order that it might be compared with the inbred lines. Nevertheless, competition within such a population will involve the interaction of numerous genotypes and these combined effects have been pooled together in the estimate of $\left(-b_{m}\right)$. Given that the population will be segregating for the genes which determine competitive ability, it is not unreasonable to assume that those individuals capable of exerting the strongest competitive pressure on their contemporaries might be favoured by selection. This would result in a high estimate of intra-genotypic competition such as we find in the present data for individuals from the Texas population.

The most obvious trend amongst the estimates of inter-genotypic competition (table 5) is the in duoculture $(0 \cdot 103431)$. In much the same way we can calculate the response of each genotype to the competitive pressure (or aggression) it faces from its associates.

The extent of the variation for aggression and response is shown clearly by an analysis of variance of the individual competitive values which reveals highly significant differences both in the overall response $\left(\chi_{8}^{2}=77 \cdot 84 ; P<0 \cdot 001\right)$ and in the overall aggression $\left(\chi_{8}^{2}=135.23 ; P<0.001\right)$ of the nine genotypes. A comparison of the two tests reveals a trend which has been pointed out by Mather and Caligari (1983). They identified pressure (or aggression) as a much more variable component of competition than response (or sensitivity). This trend is repeated in the present analysis where variation for aggression is almost twice that for response.

The relationship between these parameters can be revealed by calculating the mean aggression (taken over all indicators) and the mean response (taken over all associates) of each genotype (table

Table 5 Estimates of $\left(b_{d}-b_{m}\right)$ the inter-genotypic competitive effect of associate on indicator for each of the duocultures raised. All estimates are subject to a standard deviation of \pm 0.057524 . Tests of significance are given as in table 4

\begin{tabular}{|c|c|c|c|c|c|c|c|}
\hline Indicator & Associate & $\left(b_{d}-b_{m}\right)$ & $P$ & Indicator & Associate & $\left(b_{d}-b_{m}\right)$ & $P$ \\
\hline Population & $y^{2} T 5$ & $0 \cdot 103431$ & NS & $y^{2} T 5$ & Population & 0.453571 & $* * *$ \\
\hline Population & $y^{2} T 17$ & $0 \cdot 111764$ & NS & $y^{2} T 17$ & Population & 0.222619 & $* * *$ \\
\hline Population & $y^{2} T 19$ & -0.020378 & NS & $y^{2} T 19$ & Population & 0.155952 & $* *$ \\
\hline Population & $y^{2} T 25$ & $0 \cdot 171288$ & $* *$ & $y^{2} T 25$ & Population & 0.442857 & $* * *$ \\
\hline
\end{tabular}

difference between the inbred lines and the Texas population. Only one of the inbred lines $\left(y^{2} T 25\right)$ shows significant levels of competition against the population. Individuals from the population, however, are clearly capable of competing very strongly against all of the inbred lines. We therefore see individuals from the population as highly effective competitors, both amongst themselves and in combination with the inbred lines. Of the inbred lines sampled $y^{2} T 25$ is the strongest competitor, both intra and inter-genotypically and this presumably reflects the particular gene complement fixed within this inbred line.

Using the individual competitive values $\left(-b_{m}\right)$ and $\left(b_{d}-b_{m}\right)$ we can calculate the competitive pressure (or aggression) exerted by an associate upon an indicator. For example, the genotype $y^{2} T 25$ exerts competitive pressure both on itself in monoculture $(0 \cdot 168771)$ and on the population
6). It is clear that although both mean aggression and mean response vary, there is no indication that they do so in a correlated fashion $(r=0 \cdot 18$, $\boldsymbol{P}>0.05)$. It can also be shown that there is no correlation in the rank order of the nine genotypes with respect to each character (Spearmans rank correlation $\left.r_{s}=0.35 ; \boldsymbol{P}>0.05\right)$. The fact that there is no way of predicting mean response from mean aggression (or vice versa) suggests that these characters may be governed by separate genetic systems as inferred by Mather and Caligari (1983).

Further useful information can be obtained if we return to the separate analyses of the eight combinations of Texas population with inbred line, as described in section 2 . In each case the competitive components were separated yielding the values in table 7 .

The estimates provide confirmation that little correlation exists between aggression and response 
Table 6 Rank order of the experimental material with respect to both mean aggression (taken over all indicators) and mean response (taken over all associates). The variance of the individual competitive values ( $V c$ ) upon which these means are based amounts to 0.003481 for 64 degrees of freedom

\begin{tabular}{llllll}
\hline Rank & Genotype & Mean aggression & $P$ & Genotype & Mean response \\
\hline 1 & Pop & $0 \cdot 334131 \pm 0 \cdot 019667$ & $* * *$ & $y^{2} T 25$ & $0 \cdot 342399 \pm 0 \cdot 041719$ \\
2 & $y^{2} T 25$ & $0 \cdot 206614 \pm 0 \cdot 041719$ & $* * *$ & $y^{2} T 5$ & $0 \cdot 311171 \pm 0 \cdot 041719$ \\
3 & $y^{2} T 5$ & $0 \cdot 136101 \pm 0 \cdot 041719$ & $* *$ & $y^{2} T 27$ & $0 \cdot 302334 \pm 0 \cdot 041719$ \\
4 & $y^{2} T 28$ & $0 \cdot 095534 \pm 0 \cdot 041719$ & $*$ & $y^{2} T 28$ & $0 \cdot 260485 \pm 0 \cdot 041719$ \\
5 & $y^{2} T 15$ & $0 \cdot 061651 \pm 0 \cdot 041719$ & NS & $y^{2} T 1$ & $0 \cdot 207836 \pm 0 \cdot 041719$ \\
6 & $y^{2} T 17$ & $0 \cdot 047594 \pm 0 \cdot 041719$ & NS & $y^{2} T 15$ & $0 \cdot 164697 \pm 0 \cdot 041719$ \\
7 & $y^{2} T 27$ & $0 \cdot 045122 \pm 0 \cdot 041719$ & NS & $y^{2} T 17$ & $0 \cdot 103022 \pm 0 \cdot 041719$ \\
8 & $y^{2} T 1$ & $0 \cdot 013250 \pm 0 \cdot 041719$ & NS & Pop & $0 \cdot 073189 \pm 0 \cdot 019667$ \\
9 & $y^{2} T 19$ & $-0 \cdot 028413 \pm 0 \cdot 041719$ & NS & $y^{2} T 19$ & $0 \cdot 059752 \pm 0 \cdot 041719$ \\
\hline
\end{tabular}

Table 7 Deviations from the overall mean competitive value ascribable to aggression $(a)$, response $(r)$ and the interaction of the two $(i)$ for each of the eight combinations of Texas population (Pop) and inbred line. The standard errors (S.E.) of the estimates are given together with the number of degrees of freedom upon which the $t$ tests are based. Results of the tests of significance are given as in table 4

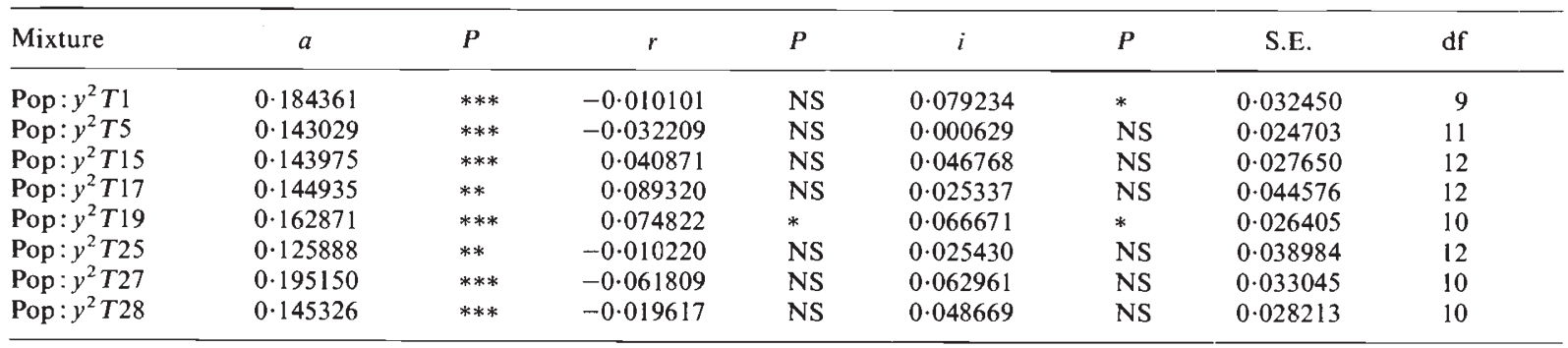

values. If the mixtures are ranked from 1 to 8 with respect to both $a$ and $r$, the relationship between the variables can be tested using Spearmans rank correlation. This yields $r_{s}=-0.095 ; P>0.05$ and gives no indication of any detectable correlation in the variation of aggression and response components. We can also consider the way in which the $a$ and $r$ components combine in their effect on the character $p_{a}$. For most of the mixtures the components combine additively with no suggestion of any significant interaction. For two of the mixtures, however, (Pop: $y^{2} T 1$ and Pop: $y^{2} T 19$ ) the components do not behave additively and the interaction component is significant. It is interesting that these two mixtures provide some evidence of facilitation rather than competition. For example, the presence of eggs of the genotype $y^{2} T 1$ actually raises the probability of a population egg giving rise to an adult fly $\left[\left(b_{d}-b_{m}\right)=-0.076331\right]$ although this effect is not statistically significant. In this respect, these results are consistent with those of Mather and Caligari (1983) who also found a departure from additivity resulting (in their case) from significant facilitation.

\section{DISCUSSION}

The results presented in this paper illustrate the complexity of the competitive interactions which take place among genotypes of Drosophila melanogaster and show how these interactions can be subjected to a satisfactory quantitative analysis. It is clear from these and from previous results that such interactions in genetically heterogeneous mixtures involve both intra and inter-genotypic effects and that these must be quantified separately in order to appreciate fully the role of a particular genotype (Caligari, 1980; Mather and Caligari, 1981; Mather et al., 1982; Mather and Caligari, 1983). Indeed, this must be true not only for Drosophila cultures but also for any group of plants or animals which are subject to competitive interaction. The need for an unambiguous quantification of these effects has been apparent for some time both in relation to agricultural practice and to genetical and ecological studies of natural populations. With respect to the former, the widespread use of mixed cropping makes an understanding of the performance of mixtures essential (Papendick et al.). Previous analyses, principally of the type 
developed by De Wit (1960), did not allow for the satisfactory separation of intra and inter-genotypic effects (Willey, 1979). This has been pointed out recently by Spitters $(1983 a, b)$ who has introduced an analysis based on the relationship between biomass and density in maize which has certain similarities to the model presented in this paper. Linear regressions of biomass per plant onto density were obtained for both single species and for mixtures of two species. The resulting slopes were then compared in order to achieve a mathematical separation of the intra and inter-specific effects.

The separation of the intra and inter-genotypic effects reported in this paper for the Texas population reveals the extent of the variation found for these characters. The inbred lines used appear to be representative of the range of variation found within the population and their inherent competitive characteristics clearly depend upon the gene complement fixed during the inbreeding programme. Individuals from the population itself were found to be very able competitors and it is thought that this must reflect the action of selective forces operating within the population.

It has been demonstrated that a distinction can be drawn between the competitive pressure that one individual can exert upon another (aggression) and the sensitivity of an individual to such competitive pressure (response). The results suggest that aggression and response behave as separately adjustable metrical characters in a manner similar to that described by Caligari and Mather (1975) with respect to mean expression and environmental sensitivity in the genotype/environment interactions of Drosophila melanogaster. They observed that the two characters were separately adjustable by selection and pointed out that this could be explained by postulating independent genetic control but that this was not a necessary conclusion. If the genes responsible for the determination of mean expression also had an effect on environmental sensitivity such that the effects on the two characters were related by some variable function, then the observed relationship between mean expression and environmental sensitivity would clearly depend upon the particular sample of genes involved in the investigation. In this way it was shown that the two characters could be separately adjusted by the selection of particular groups of genes.

A similar form of genetic control may be involved in the present study with respect to aggression and response. The data may suggest an independent genetic control but it is also possible to envisage a situation where the genes which determine aggression are involved in the control of response in such a way that the effect on the two characters is related but not in any systematic fashion. The actual relationship observed between aggression and response would therefore be variable and would depend on the genotypes involved. Whether aggression and response are determined in this way (i.e., by uncorrelated pleiotropic effects of a single set of genes) or by two distinct groups of genes, the results of the present study make it clear that they are separately adjustable by selection. The sample of genotypes employed in this experiment, for example, displays all possible combinations of high and low levels of mean aggression and response. The extreme combinations are shown in table 8 although other genotypes provide

Table 8 Examples of genotypes displaying various combinations of high $(\mathrm{H})$ and low (L) levels of mean aggression and mean response

\begin{tabular}{cll}
\hline Mean aggression & Mean response & Example genotype \\
\hline$(0.206614) \mathrm{H}$ & $\mathrm{H}(0.342399)$ & $y^{2} T 25$ \\
$(0.334131) \mathrm{H}$ & $\mathrm{L}(0.073189)$ & Population \\
$(0.045122) \mathrm{L}$ & $\mathrm{H}(0.302334)$ & $y^{2} T 27$ \\
$(-0.028413) \mathrm{L}$ & $\mathrm{L}(0.059752)$ & $y^{2} T 19$ \\
\hline
\end{tabular}

intermediate combinations. Thus, the particular gene complement of $y^{2} T 19$ results in an inability to exert detectable levels of aggression against other genotypes, whilst on the other hand it is little affected by the competitive pressure it must face in mixed culture. Conversely, $y^{2} T 25$ exerts high levels of aggression but it is also very sensitive to competitive pressure from other genotypes. The other examples given in table 8 show that high levels of aggression do not necessarily result in high levels of response and vice versa.

A further point concerns the behaviour of the Texas population with respect to aggression and response. In any genetically segregating population it is reasonable to assume that selection might favour increased levels of aggression between larvae in competitive situations. The results of this investigation indicate that such selection has been operative within the Texas population in respect of the character $p_{a}$, producing an average level of aggression $(0.334131)$ well in excess of that of even the best inbred line $(0 \cdot 206614)$. If this is a true reflection of the situation then every developing individual within the population will be subjected to generally high levels of aggression. Under these conditions, only one strategy would result in a competitive advantage for any one genotype within 
the population. This would be a progressive reduction in the sensitivity of that genotype to the prevailing high levels of aggression. Thus we can envisage a situation where selection favours increased levels of aggression on the one hand and decreased levels of response on the other. This hypothesis, of course, can only sensibly be developed in respect of the situation where aggression and response are separately adjustable by selection. The results of this investigation suggest that a dual selective system of this kind may well be operative within the Texas population which has, on average, the highest levels of aggression and (with the exception of $y^{2} T 19$ ) the lowest levels of response.

Clearly, this form of analysis provides a flexible approach to the study of competition. In the present example the interactions observed for the character $p_{a}$ in Drosophila must depend upon factors such as larval feeding rate, critical weights, food conversion efficiency, the secretion and excretion of various substances into the medium and even the exploitation of behavioural characteristics. The specific involvement of such biological processes, however, must await further investigation.

Acknowledgements I would like to express my gratitude to Professor Sir Kenneth Mather for his advice and for reading and commenting on the original manuscript. I am also indebted to Dr P. D. S. Caligari both for helpful discussion and for his kind donation of the yellow marked inbred lines.

\section{REFERENCES}

BREESE, E. L. AND HILL, J. 1973. Regression analysis of interactions between competing species. Heredity, 31, 181-200.

CALIGARI, P. D. S. 1980 . Competitive interactions in Drosophila melanogaster. I. Monocultures. Heredity, 45, 219-231.

CALIGARI, P. I). S. AND MATHER, K. 1975. Genotype-environment interaction III. Interactions in Drosophila melanogaster. Proc, R. Soc. Lond. B., 191, 387-411.

FISHER, R. A. AND YATES, F. 1963. Statistical Tables for Biological, Agricultural and Medical Research, 6th edn. Oliver and Boyd, Edinburgh.

LINNEY, R. BARNES, B. W. AND KEARSEY, M. J. 1971. Variation for metrical characters in Drosophila populations. Heredity, $27,163-174$

MATHER, K. AND CALIGARI, P. D. S. 1981. Competitive interactions in Drosophila melanogaster. II. Measurement of competition. Heredity, 46, 239-254.

MATHER, K. AND CALIGARI, P. D. S. 1983. Pressure and tesponse in competitive interactions. Heredity, 51, 435-454.

MATHER, K., HILl, J. AND CALIGARI, P. I). S. 1982. Analysis of competitive ability among genotypes of perennial ryegrass. Heredity, $48,421-434$

PAPENDICK, R. I. 1976. In Sanchez, P. A. and Triplett, G. B. (eds.) Multiple Cropping, American Society of Agronomy, Special Publication, 27, $378 \mathrm{pp}$.

SPITTERS, C. J. T. $1983 a$. An alternative approach to the analysis of mixed cropping experiments. 1. Estimation of competition effects. Neth, J. agric. Sci., 31, 1-11.

SPITTERS. C. J. T. $1983 \mathrm{~b}$. An alternative approach to the analysis of mixed cropping experiments. 2. Marketable yield. Neth. J. agric. Sci., 31, 143-155.

WILLEY, R. W. 1979. Intercropping-its importance and research needs. Field Crop Abstr., 32, 1-10, 73-85.

WIT, C. T. DE. 1960. On competition. Versl. Landbouwk. Onderz. Ned., 66, 1-82. 